\title{
Thrombolysis in myocardial infarction (TIMI) risk score validation in Saudi Arabia
}

\author{
Saad Al-Bugami, Faisal Al-Husayni, Samer Alamri, Rakan Aljedaani, Mohammad Zwawy, Abdulaziz Al-Zahrani
}

\begin{abstract}
Al-Bugami S, Al-Husayni F, Alamri S, et al. Thrombolysis in myocardial infarction (TIMI) risk score validation in Saudi Arabia. Curr Res Cardiol 2018;5(1):4-8.

BACKGROUND: Early risk stratification plays a pivotal role in the optimal management of non-ST segment elevation myocardial infarction/unstable angina (NSTEMI/UA) acute coronary syndromes and certainly improving patients care and their final outcomes. Thrombolysis in myocardial infarction (TIMI) risk score was developed from randomized clinical trials on patients with NSTEMI and UA, and it has been validated in non-selected Western patient populations.
\end{abstract}

OBJECTIVE: The aim of the present study was to assess the validity of TIMI risk score on NSTEMI and UA patients in King Abdulaziz Medical City, Jeddah, Saudi Arabia.

METHODS: This cross-sectional study was undertaken on 194 patients diagnosed with NSTEMI or UA. They were consecutively included over a two-year period. Data were collected from medical records, TIMI score was

Cardiovascular diseases are the leading cause of morbidity and mortality globally and in the Middle East (1). The major cause of cardiovascular mortality is coronary artery disease (2). Acute coronary syndrome is the major presentation of coronary artery disease, and it includes a spectrum of disorders like unstable angina (UA), ST elevation myocardial infarction (STEMI) and non-ST elevation myocardial infarction (NSTEMI) (3).

Patients presenting with an acute coronary syndrome without ST segment elevation are diagnosed as having unstable angina or non-ST elevation myocardial infarction (UA/NSTEMI). Such patients are at high risk for death, myocardial infarction or severe recurrent cardiac ischemic events (4).

Careful risk assessment of these patients helps clinicians in decision making regarding therapeutic interventions, triage among alternative levels of hospital care (e.g. intensive care unit vs hospital ward vs outpatient care), and allocation of clinical resources. Additionally, prognostication of patient risk helps in providing valuable information for patients and their families (5).

Several multivariable risk models for UA/NSTEMI have been developed to help physicians risk stratify patients in a standardized and uniform manner. Thrombolysis in myocardial infarction (TIMI) risk score was developed from multi-center international randomized clinical trials on patients with NSTEMI and UA, and it has been validated in non-selected Western patient populations. It is simple, low cost, easily applicable risk score using standard patient features that could be readily identified at presentation (6-9).

The TIMI risk score was shown to provide good discrimination in predicting in hospital and 1-year mortality (10). This offers some evidence for its clinical applicability in risk stratification and prognostication. However, it is not known how the TIMI risk score performs in a population with many characteristic differences from the TIMI population in which it was derived from. Hence, the aim of the current study was to assess the validity of TIMI risk score on NSTEMI and UA patients in King Abdulaziz Medical City, Jeddah, Saudi Arabia.

\section{METHODS}

This is a retrospective cross sectional single center study conducted at King calculated and 30 days outcome was recorded. Model discrimination and calibration was tested in the overall enrolled population.

RESULTS: Validity of TIMI score in predicting in hospital mortality within 30 days of diagnosis of NSTEMI or UA in Saudi population was assessed by ROC curve and binary logistic regression analysis. The accuracy of discrimination of the TIMI risk score was poor; the area under the ROC curve (AUC) or C-index was 0.612 (95\% CI: 0.539 to 0.681). Binary logistic regression model revealed good calibration or model fit of TIMI risk score as revealed by the Hosmer and Lemes how test for the constructed logistic model; p value was 0.934 (>0.05) denoting good model fit.

CONCLUSION: The generalizability of this single-center study requires further confirmation in a larger sample population allowing stratification of the study participants into different subgroups according to known important risk factors such as diabetes mellitus, renal failure, or age, so that the performance of the TIMI risk score could be comprehensively assessed before deciding its application in our population.

Key Words: TIMI risk score; Acute coronary syndrome; Validation

Faisal Cardiac Center in Jeddah, Saudi Arabia. Consecutive patients with the diagnosis of acute coronary syndrome excluding STEMI from August 2014 till August 2016 have been included. Exclusion criteria included overt heart failure, severe left ventricular dysfunction, cardiogenic shock, hemodynamic instability and unstable ventricular arrhythmia as well as Patients with recurrent or persistent rest angina despite intensive medical therapy and those not eligible for coronary catheterization.

The data were collected from the medical records using pre-designed data collection sheet, which included Patients' characteristics. TIMI score parameters. Patients' outcomes within 30 days (in hospital mortality, readmission, CABG, Percutaneous Coronary Intervention).

\section{Data management and statistical analysis}

TIMI score (9) has seven clinical parameters, each one counts for one point. These parameters are age $\geq 65$, three or more coronary artery disease risk factors, known coronary artery disease (Coronary stenosis $\geq 50 \%$ ), acetyl salicylic acid (ASA) use in the past seven days, severe angina ( $\geq 2$ episodes in 24 hours), electrocardiogram (ECG) ST changes $(0.5 \mathrm{~mm})$, and positive cardiac biomarkers

For categorical variables, frequencies and percentages were reported. Differences between groups (in-hospital mortality) were analyzed using Pearson Chi-Square tests (or Fisher's exact test for cells less than 5). For continuous normally distributed variables, mean $\pm \mathrm{SD}$ were presented, and independent $\mathrm{T}$ test was conducted. For normally distributed continuous variables, medians and interquartile ranges (IQR) were presented and analyses were conducted using Mann-Whitney test. The association between in-hospital mortality and readmission (outcome variables) and the TIMI risk score (predictor variable) was assessed using Fisher's exact test.

A different multivariate logistic regression model was also constructed to evaluate the performance of the 7 TIMI predictors with in-hospital mortality as the outcome variable.

Validation of TIMI score was done by the goodness-of-fit of the logistic model which was examined using the Hosmer and Lemeshow goodness-of-

Department of Cardiology, King Saud Bin Abdulaziz University for Health Sciences, King Abdulaziz Medical City, Jeddah, Saudi Arabia

Correspondence: Dr Saad Al-Bugami, Department of Cardiology, King Saud Bin Abdulaziz University for Health Sciences, King Abdulaziz Medical City, Jeddah 21423, Saudi Arabia, Telephone 00966505516952, email Bogamisa@ngha.med.sa, sbugami@gmail.com

Received: January 23, 2018, Accepted: February 07, 2018, Published: February 12, 2018

open Access

This open-access article is distributed under the terms of the Creative Commons Attribution Non-Commercial License (CC BY-NC) (http:// creativecommons.org/licenses/by-nc/4.0/), which permits reuse, distribution and reproduction of the article, provided that the original work is properly cited and the reuse is restricted to noncommercial purposes. For commercial reuse, contact reprints@pulsus.com 
fit statistic (11), which analyzes the actual versus the predicted responses; theoretically, the observed and expected counts should be close. Based on the $\mathrm{x}^{2}$ distribution, a Hosmer and Lemeshow statistic with a p-value greater than 0.05 is considered a good fit. The discriminatory power of the score was assessed by the area under the receiver operative characteristics (ROC) curve also known as $\mathrm{C}$-index (12). A model with perfect discriminative ability has a C-index of 1.0; an index of 0.5 provides no better discrimination than chance. Models with area under the ROC curve of greater than 0.7 are preferred. Statistical analyses were conducted using SPSS version $20 \mathrm{P}<0.05$ was considered statistically significant.

\section{Ethical considerations}

All procedures performed in this study were in accordance with the ethical standards of the institutional research committee and with the 1964 Helsinki declaration and its later amendments. Only patients' medical records were reviewed (informed consent was not required). Patients' files were approached in the Medical Record Department. The study met all institutional ethical board requirements.

\section{RESULTS}

This study was undertaken on 194 patients diagnosed as NSTEMI ( $=122)$ and UA $(\mathrm{N}=72)$. Table 1 shows demographics, clinical characteristics, and TIMI score of the studied patients stratified by in hospital mortality. The ages ranged from 24 to 99 years with a mean of $64.43 \pm 14.19$. Males were predominant $(68.0 \%)$. Total of nineteen patients $(9.8 \%)$ died ten patients $(5.2 \%)$ died during index hospitalization in the medically treated group who were thought not amenable to revascularization and nine patients $(4.6 \%)$ died within thirty days of coronary intervention. Compared to survivors, patients who died were insignificantly older (mean age=68.11 \pm 12.65 ), mainly males $(57.9 \%)$, more likely to have cardiovascular risk factors $(73.7 \%)$ and two or more anginas in the last 24 hours. In addition, statistically higher percentage $(73.7 \%)$ of them had elevated cardiac biomarkers and did not use acetyl salicylic acid in the last 7 days. The median TIMI risk score in all of the studied patients was 3 (IQR: 2-4). No statistically significant difference was found between survivors and non-survivors regarding the median TIMI risk score $(\mathrm{p}=0.099)$.

The frequency of percutaneous Coronary Intervention (PCI) in all patients was $68.8 \%$; the thirty-day mortality was $4.6 \%$ and the readmission was $3.6 \%$. Ten cases (5.1\%) underwent CABG; one patient required readmission. Twenty-one patients (10.8\%) had subsequent CABG following initial PCI. The total number of patients who needed readmission to hospital was 13 (6.7\%).

Patient's outcomes within 30 days of NSTEMI or UA diagnosis are shown in table 2.

Table 3 demonstrates statistically significant association between the increasing TIMI risk score and the frequency of readmission to hospital $(\mathrm{p}=0.011)$.

Higher percent of patients (69.2\%) had TIMI score of 4. On the other hand, TIMI risk score did not show statistically significant association with in hospital mortality $(\mathrm{p}=0.304)$.

Validity of TIMI score in predicting in hospital mortality within 30 days of diagnosis of NSTEMI or UA in Saudi population was assessed by ROC curve and binary logistic regression analysis. The accuracy of discrimination of the

\section{TABLE 1}

Demographics, clinical characteristics and thrombolysis in myocardial infarction (TIMI) score of the studied patients

\begin{tabular}{|c|c|c|c|c|c|c|c|}
\hline \multirow{4}{*}{ Variables } & & & \multicolumn{3}{|c|}{ In hospital mortality } & \multirow{4}{*}{ Test statistic ${ }^{\star *}$} & \multirow{4}{*}{$\mathbf{p}$} \\
\hline & & & \multicolumn{3}{|c|}{ During index admission } & & \\
\hline & & & \multirow{2}{*}{$\begin{array}{c}\text { Yes } \\
\mathrm{N}=19(9.8 \%)\end{array}$} & \multirow{2}{*}{$\begin{array}{c}\text { No } \\
N=175(90.2 \%)\end{array}$} & \multirow{2}{*}{$\begin{array}{c}\text { Total } \\
\mathrm{N}=194(100 \%)\end{array}$} & & \\
\hline & & & & & & & \\
\hline \multirow{2}{*}{ Age } & \multicolumn{2}{|c|}{ Range } & $40.00-84.00$ & $24.00-99.00$ & $24.00-99.00$ & \multirow{2}{*}{-1.89} & \multirow{2}{*}{0.236} \\
\hline & \multicolumn{2}{|c|}{ Mean \pm SD } & $68.11 \pm 12.65$ & $64.03 \pm 14.33$ & $64.43 \pm 14.19$ & & \\
\hline \multirow{4}{*}{ Gender } & \multirow{2}{*}{ Male } & $\mathrm{N}$ & 11 & 121 & 132 & \multirow{4}{*}{0.997} & \multirow{4}{*}{0.318} \\
\hline & & $\%$ & $57.90 \%$ & $69.10 \%$ & $68.00 \%$ & & \\
\hline & \multirow{2}{*}{ Female } & $\mathrm{N}$ & 8 & 54 & 62 & & \\
\hline & & $\%$ & $42.10 \%$ & $30.90 \%$ & $32.00 \%$ & & \\
\hline \multirow{4}{*}{ Cardiovascular disease risk factors } & \multirow{2}{*}{ Yes } & $\mathrm{N}$ & 14 & 101 & 115 & \multirow{4}{*}{1.811} & \multirow{4}{*}{0.178} \\
\hline & & $\%$ & $73.70 \%$ & $57.70 \%$ & $59.30 \%$ & & \\
\hline & \multirow{2}{*}{ No } & $\mathrm{N}$ & 5 & 74 & 79 & & \\
\hline & & $\%$ & $26.30 \%$ & $42.30 \%$ & $40.70 \%$ & & \\
\hline \multirow{4}{*}{ Acetyl salicylic acid use in last 7 days } & \multirow{2}{*}{ Yes } & $\mathrm{N}$ & 5 & 117 & 122 & & \\
\hline & & $\%$ & $26.30 \%$ & $66.90 \%$ & $62.90 \%$ & 1207 & $0001^{*}$ \\
\hline & \multirow{2}{*}{ No } & $\mathrm{N}$ & 14 & 58 & 72 & 12.01 & $0.00 T^{\circ}$ \\
\hline & & $\%$ & $73.70 \%$ & $33.10 \%$ & $37.10 \%$ & & \\
\hline & & $\mathrm{N}$ & 13 & 99 & 112 & & \\
\hline & Yes & $\%$ & $68.40 \%$ & $56.60 \%$ & $57.70 \%$ & & \\
\hline 2 or more anginas in last 24 hours & & $\mathrm{N}$ & 6 & 76 & 82 & 0.986 & 0.321 \\
\hline & No & $\%$ & $31.60 \%$ & $43.40 \%$ & $42.30 \%$ & & \\
\hline & Yoc & $\mathrm{N}$ & 7 & 78 & 85 & & \\
\hline ST change $(05 \mathrm{~mm})$ & & $\%$ & $36.80 \%$ & $44.60 \%$ & $43.80 \%$ & 0116 & 510 \\
\hline si crange (0.5 mm) & $\mathrm{No}$ & $\mathrm{N}$ & 12 & 97 & 109 & 0.410 & 0.519 \\
\hline & INO & $\%$ & $63.20 \%$ & $55.40 \%$ & $56.20 \%$ & & \\
\hline & Yec & $\mathrm{N}$ & 5 & 32 & 37 & & \\
\hline Known coronary artery disease ( $>50 \%$ stenosis) & & $\%$ & $26.30 \%$ & $18.30 \%$ & $19.10 \%$ & 029 & 0283 \\
\hline 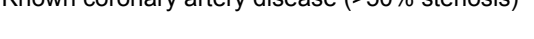 & $\mathrm{No}$ & $\mathrm{N}$ & 14 & 143 & 157 & 0.20 & 0.200 \\
\hline & No & $\%$ & $73.70 \%$ & $81.70 \%$ & $80.90 \%$ & & \\
\hline & Yes & $\mathrm{N}$ & 14 & 44 & 58 & & \\
\hline Flevated cardiac markers & Yes & $\%$ & $73.70 \%$ & $25.10 \%$ & $29.90 \%$ & & \\
\hline Elevated cardiac markers & & $\mathrm{N}$ & 5 & 131 & 136 & 19.269 & $<0.001^{\star}$ \\
\hline & No & $\%$ & $26.30 \%$ & $74.90 \%$ & $70.10 \%$ & & \\
\hline & Ran & & $1.00-6.00$ & $1.00-6.00$ & $1.00-6.00$ & & \\
\hline TIMUL cooro & Medi & & 4 & 3 & 3 & 1610 & 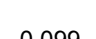 \\
\hline Mivil score & IQF & & $3.00-5.00$ & $2.00-4.00$ & $2.00-4.00$ & -1.049 & 0.099 \\
\hline & Mean & & 117.08 & 95.37 & - & & \\
\hline
\end{tabular}


TABLE 2

In hospital and thirty days outcome

\begin{tabular}{|c|c|c|c|c|c|c|c|}
\hline & \multirow{3}{*}{ Variables } & & \multicolumn{3}{|c|}{ Studied patients } & \multicolumn{2}{|c|}{ Fisher's Exact test } \\
\hline & & & \multirow{2}{*}{$\begin{array}{l}\text { NSTEMI } \\
\mathrm{N}=122\end{array}$} & \multirow{2}{*}{$\begin{array}{c}\text { Unstable angina } \\
\qquad \mathrm{N}=72\end{array}$} & \multirow{2}{*}{$\begin{array}{c}\text { Total } \\
N=194\end{array}$} & \multirow{2}{*}{$\mathbf{X}^{2}$} & \multirow{2}{*}{ p value } \\
\hline & & & & & & & \\
\hline \multicolumn{8}{|c|}{ In hospital outcomes } \\
\hline & \multirow{2}{*}{ Percutaneous Coronary Intervention } & $\mathrm{N}$ & 71 & 35 & 106 & & \\
\hline & & $\%$ & 58.2 & 48.6 & 54.6 & & \\
\hline & \multirow{2}{*}{ CABG } & $\mathrm{N}$ & 1 & 8 & 9 & & \\
\hline & & $\%$ & 0.8 & 11.1 & 4.6 & & \\
\hline & \multirow{2}{*}{ In hospital mortality for medically treated patient } & $\mathrm{N}$ & 4 & 6 & 10 & & \\
\hline & & $\%$ & 3.3 & 8.3 & 5.2 & & \\
\hline & \multirow{2}{*}{ Medical Treatment/ improvement } & $\mathrm{N}$ & 22 & 6 & 28 & & \\
\hline & & $\%$ & 18 & 8.3 & 14.4 & & \\
\hline & Thirty-c & om & & & & & \\
\hline \multirow{12}{*}{ Outcome } & \multirow{2}{*}{$\mathrm{PCl}$ and Subsequent $\mathrm{CABG}$} & $\mathrm{N}$ & 15 & 4 & 19 & & \\
\hline & & $\%$ & 12.3 & 5.6 & 9.8 & 31.042 & $<0.001^{*}$ \\
\hline & \multirow{2}{*}{ Readmission following PCl } & $\mathrm{N}$ & 4 & 3 & 7 & & \\
\hline & & $\%$ & 3.3 & 4.2 & 3.6 & & \\
\hline & \multirow{2}{*}{ Readmission following $\mathrm{PCl}$ and Subsequent $\mathrm{CABG}$} & $\mathrm{N}$ & 1 & 1 & 2 & & \\
\hline & & $\%$ & 0.8 & 1.4 & 1 & & \\
\hline & \multirow{2}{*}{$\mathrm{PCI} 30$ days Mortality } & $\mathrm{N}$ & 1 & 8 & 9 & & \\
\hline & & $\%$ & 0.8 & 11.1 & 4.6 & & \\
\hline & \multirow{2}{*}{ Re admission (for medically treated patients) } & $\mathrm{N}$ & 3 & 0 & 3 & & \\
\hline & & $\%$ & 2.5 & 0 & 1.5 & & \\
\hline & \multirow{2}{*}{ Readmission following CABG } & $\mathrm{N}$ & 0 & 1 & 1 & & \\
\hline & & $\%$ & 0 & 1.4 & 0.5 & & \\
\hline
\end{tabular}

${ }^{*}$ Significant at $p<0.05$, CABG: coronary artery bypass graft

TABLE 3

Association between thrombolysis in myocardial infarction (TIMI) score and hospital readmission and in hospital mortality within 30 days of diagnosis of non- ST segment elevation myocardial infarction and unstable angina

\begin{tabular}{|c|c|c|c|c|c|c|c|c|c|c|c|}
\hline & & & Read & issi & & & hospit & mo & tality & & \\
\hline & & & es & & lo & & es & & No & & \\
\hline Variabl & & & $\begin{array}{l}=13 \\
7 \%)\end{array}$ & & $\begin{array}{l}181 \\
.3 \%)\end{array}$ & & $\begin{array}{l}=19 \\
8 \%)\end{array}$ & & $\begin{array}{l}175 \\
.2 \%)\end{array}$ & & $\begin{array}{l}194 \\
0 \%)\end{array}$ \\
\hline & & $\mathbf{N}$ & $\%$ & $\mathbf{N}$ & $\%$ & $\mathbf{N}$ & $\%$ & $\mathbf{N}$ & $\%$ & $\mathbf{N}$ & $\%$ \\
\hline & 1 & 1 & 7.7 & 13 & 7.2 & 1 & 5.3 & 13 & 7.4 & 14 & 7.2 \\
\hline & 2 & 3 & 23.1 & 36 & 19.9 & 2 & 10.5 & 37 & 21.1 & 39 & 20.1 \\
\hline TIMI & 3 & 0 & 0 & 56 & 30.9 & 5 & 26.3 & 51 & 29.1 & 56 & 28.9 \\
\hline score & 4 & 9 & 69.2 & 47 & 26 & 6 & 31.6 & 50 & 28.6 & 56 & 28.9 \\
\hline & 5 & 0 & 0 & 24 & 13.3 & 3 & 15.8 & 21 & 12 & 24 & 12.4 \\
\hline & 6 & 0 & 0 & 5 & 2.8 & 2 & 10.5 & 3 & 1.7 & 5 & 2.6 \\
\hline Fisher's & $x^{2}$ & & & & & & & & 519 & & \\
\hline $\begin{array}{c}\text { Exact } \\
\text { test }\end{array}$ & $\mathrm{p}$ & & & & & & & & 304 & & \\
\hline
\end{tabular}

TIMI risk score was poor; the area under the ROC curve (AUC) or C-index was 0.612 (95\% CI: 0.539 to 0.681) as illustrated in Figure 1.

Binary logistic regression model revealed good calibration or model fit of TIMI risk score on Saudi population as revealed by the Hosmer and Lemeshow test for the constructed logistic model; p-value was $0.934(>0.05)$ denoting good model fit. The calibration graph of the observed versus predicted in hospital mortality for all the studied patients was outlined in Figure 2.

The graph demonstrates good agreement between the observed and predicted in hospital mortality.

Table 4 demonstrates absence of statistically significant association between the likelihood of in-hospital mortality and the various TIMI score predictors as revealed by multivariate logistic regression analysis $(p>0.05)$. Only elevated cardiac markers contributed significantly to the model $(\mathrm{p}=0.025)$ where elevated cardiac biomarkers on admission increased the odds of in hospital mortality by a factor of 5.304 .

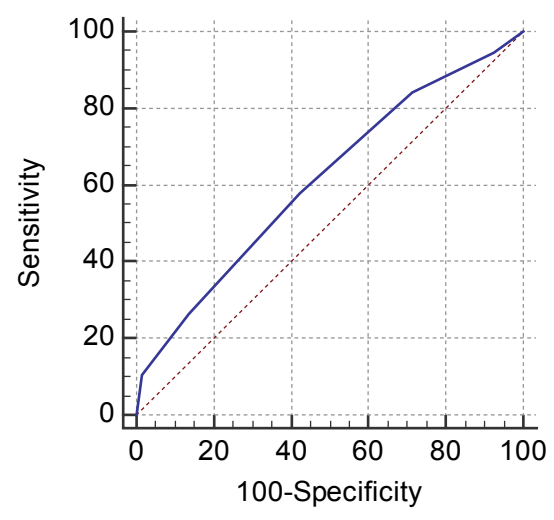

Figure 1) ROC curve for prediction of in hospital mortality within 30 days of diagnosis of non-ST segment elevation myocardial infarction and unstable angina by thrombolysis in myocardial infarction score

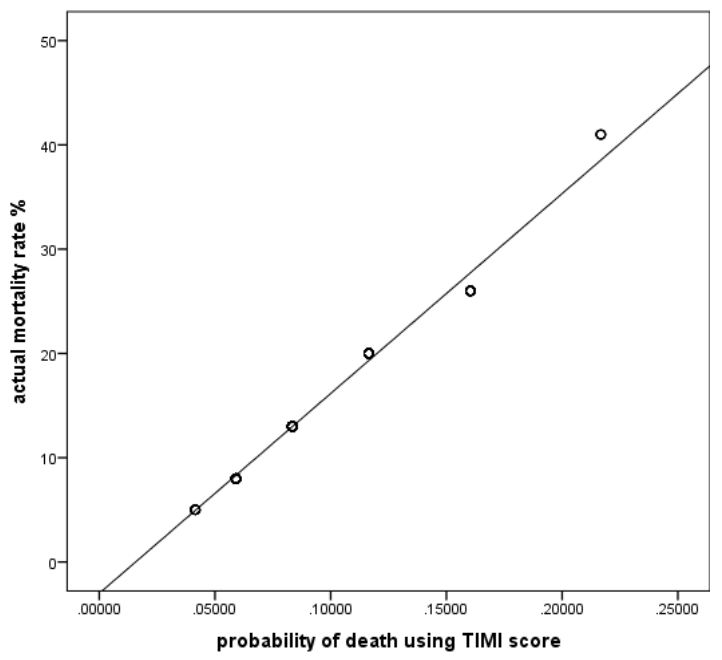

Figure 2) Calibration of in hospital mortality using deciles of predicted risk by thrombolysis in myocardial infarction (TIMI) score. The diagonal line indicates perfect calibration 


\section{TABLE 4}

Multivariable logistic regression model on the thrombolysis in myocardial infarction score variables predicting in hospital mortality

\begin{tabular}{|c|c|c|c|c|}
\hline \multirow{2}{*}{ Variables } & \multirow{2}{*}{ Odds ratio } & \multicolumn{2}{|c|}{ 95\% C.I. } & \multirow{2}{*}{$p$ value } \\
\hline & & Lower & Lower & \\
\hline Age & 1.019 & 0.975 & 1.066 & 0.394 \\
\hline Cardiovascular Risk factors & 0.807 & 0.231 & 2.824 & 0.738 \\
\hline $\begin{array}{c}\text { Acetyl salicylic acid use in last } 7 \\
\text { days }\end{array}$ & 0.324 & 0.078 & 1.354 & 0.122 \\
\hline 2 or more anginas in last 24 hours & 1.036 & 0.336 & 3.19 & 0.951 \\
\hline ST change $(0.5 \mathrm{~mm})$ & 0.479 & 0.155 & 1.479 & 0.201 \\
\hline $\begin{array}{c}\text { Known coronary artery disease } \\
(>50 \% \text { stenosis })\end{array}$ & 2.375 & 0.687 & 8.212 & 0.172 \\
\hline Elevated cardiac markers & 5.304 & 1.237 & 22.743 & $0.025^{*}$ \\
\hline
\end{tabular}

\section{DISCUSSION}

Early risk stratification plays a pivotal role in the optimal management of NSTEMI/UA acute coronary syndromes and certainly improves patients' care and their final outcomes (13). There is a growing interest in using risk scores to facilitate risk assessment (14). TIMI risk score was derived from clinical trial populations and was validated internally and externally in some populations (9). It has been compared with other risk scores and has consistently been found to have good performance and, more importantly, it can be used to predict in hospital mortality, which was the main outcome in our population (10). Due to the presence of differences between the TIMI cohort in the clinical trials from which it was developed and our population, it was necessary to be validated to determine its generalizability and applicability on Saudi population (14). In this study, the performance of TIMI risk score in predicting 30 days in hospital mortality was assessed. It revealed poor ability to accurately rank individuals from low to high risk (poor discrimination). Additionally, binary logistic regression analysis revealed good calibration or model fit of TIMI risk score on Saudi population as revealed by the Hosmer and Lemeshow test. Previous studies revealed good but lower discrimination of TIMI score when compared with other risk scores. Singh et al. (15) showed that the TIMI risk score was inferior to the Predicting Risk of Death in Cardiac Disease Tool (PREDICT) score in predicting death among 717 patients with non-ST elevation myocardial infarction in Olmsted County. In addition, de Arau 'jo Goncalves et al. (16) and Yan et al. (10) demonstrated stronger discrimination of both Integrilin Therapy risk score (PURSUIT RS) and Global Registry of Acute Cardiac Events Risk Score (GRACE RS) than TIMI risk score of the risk of death among patients presenting with wide range of acute coronary syndromes. This was attributed to absence of powerful prognostication variables like hemodynamic parameters in TIMI risk score when it was initially developed. Furthermore, the TIMI risk score composed of dichotomous variables only and with a limited range of $0-7$. This might limit its predictive accuracy (17).

The poor discrimination of TIMI in this study population is probably due to the small sample size of 194 patients in addition to the unselected convenient sample, which comprised a broad spectrum of patients. Alternatively, TIMI risk score was developed from cohort of patients included in clinical trials in which high risk patients with renal failure were excluded. This difference between the two cohorts could impact the score performance and its power of discrimination (18).

When multivariable logistic regression analysis was carried out using the seven risk predictors derived from TIMI risk score for the inhospital mortality, the model demonstrated absence of statistically significant association between various parameters and the likelihood of in hospital mortality $(\mathrm{p}>0.05)$.

\section{CONCLUSION}

In the present study, mortality was seen more among patients with TIMI risk score of 3 or 4 , whereas those who ranked higher scores were more frequently alive with no significant association between the increasing TIMI risk score and the in-hospital mortality. It should be realized that the risk scores are important clinical tools but have not included all risk factors in any particular individual. Risk scores are guides that are applicable in the majority of patients but cannot completely replace clinical judgment.

The generalizability of this single-center study requires further confirmation in a larger sample population so that the performance of the TIMI risk score could be comprehensively assessed before deciding its application to our population.

\section{POTENTIAL CONFLICTS OF INTEREST}

The authors declare that there are no conflicts of interest

\section{LIMITATIONS}

This study has many limitations that should be considered when making conclusions the study is retrospective, single center and small size but this represent real live patients and daily practice in a busy center in Saudi Arabia. Larger sized sample and consideration of individual risk factors should be looked at in future study to develop a risk model that can be applied to our population.

\section{ACKNOWLEDGEMENT}

We would like to thank Alwaleed Althobaiti, Tasneem Balkhoyor, Fatmah Alkahtani and Samah Almatrafi for their support and contribution in data collection and data entry. We would like to acknowledge the assistance provided by medical records department at King Abdulaziz Medical City for their support and cooperation.

\section{REFERENCES}

1. World Health Organization. Updated fact sheet on cardiovascular diseases, WHO. 2017.

2. Sanchis-Gomar F, Perez-Quilis C, Leischik R, et al. Epidemiology of coronary heart disease and acute coronary syndrome. Ann Translational Med 2016;4(13):256.

3. Lippi G, Sanchis-Gomar F, Cervellin G. Chest pain, dyspnea and other symptoms in patients with type 1 and 2 myocardial infarction. A literature review. Int J Cardiol 2016;215:20-2.

4. Engel J, van der Wulp I, de Bruijne M, et al. A cross-sectional multicentre study of cardiac risk score use in the management of unstable angina and non-ST-elevation myocardial infarction. BMJ Open 2015;5(11):e008523.

5. Morrow DA, Antman EM, Charlesworth A, et al. TIMI risk score for ST-Elevation Myocardial Infarction: A convenient, bedside, clinical score for risk assessment at presentation: An intravenous nPA for treatment of infarcting myocardium early II trial substudy. Circulation 2000; 102:2031-7.

6. Boersma E, Pieper KS, Steyerberg EW, et al. Predictors of outcome in patients with acute coronary syndromes without persistent ST-segment elevation. Results from an international trial of 9461 patients. The PURSUIT investigators. Circulation 2000;101:2557-67.

7. Holper EM, Antman EM, McCabe CH, et al. A simple, readily available method for risk stratification of patients with unstable angina or non- $Q$ myocardial infarction: a TIMI 11B sub study. Circulation 1998;98:I-493.

8. Antman EM, McCabe CH, Gurfinkel EP, et al. Enoxaparin prevents death and cardiac ischemic events in unstable angina/non-Q-wave myocardial infarction: results of the Thrombolysis in Myocardial Infarction (TIMI) 11B trial. Circulation 1999;100:1593-601.

9. Antman EM, Cohen M, Bernink PJ, et al. The TIMI risk score for unstable angina/non-ST elevation MI: a method for prognostication and therapeutic decision making. JAMA 2000;284:835-42.

10. Yan AT, Yan RT, Tan M, et al. Risk scores for risk stratification in acute coronary syndromes: useful but simpler is not necessarily better. Eur heart J 2007;28:1072-8.

11. Lemeshow S, Hosmer DWJ. A review of goodness of fit statistics for use in the development of logistic regression models. Am J Epidemiol 1982;115:92-106.

12. Hanley JA, McNeil BJ. The meaning and use of the area under a receiver operating characteristic (ROC) curve. Radiology 1982;143:29-36.

13. Fitchett DH, Borgundvaag B, Cantor W, et al. Non-ST segment elevation acute coronary syndromes: A simplified risk-orientated algorithm. Can J Cardiol 2006;22:663-77.

14. Steyerberg EW. Clinical prediction models: A practical approach to development, validation, and updating. New York: Springer 2010;270-9.

15. Singh M, Reeder GS, Jacobsen SJ, et al. Scores for post-myocardial infarction risk stratification in the community. Circulation 2002;106:2309-14. 
16. de Araujo Goncalves P, Ferreira J, Aguiar C, et al. TIMI, PURSUIT, and GRACE risk scores: sustained prognostic value and interaction with revascularization in NSTE-ACS. Eur Heart J 2005;26:865-72.
17. Weintraub W. Prediction scores after myocardial infarction. Value, limitations, and future directions. Circulation 2002;106:2292-3.

18. Go J, Narmi A, Sype J, et al. Impact of renal dysfunction on the prognostic value of the TIMI risk score in patients with non-ST elevation acute coronary syndrome. Coron Artery Dis 2011;22:411-5. 